CERN-PH-EP/2005-048

14 October 2005

\title{
Measurement of Nucleon Structure Functions in Neutrino Scattering
}

\author{
CHORUS Collaboration
}

\begin{abstract}
After completion of the data taking for the $\nu_{\mu} \rightarrow \nu_{\tau}$ oscillation search, the CHORUS lead-scintillator calorimeter was used in the 1998 run as an active target. High-statistics samples of charged-current interactions were collected in the CERN SPS west area neutrino beam. This beam contained predominantly muon (anti-)neutrinos from sign-selected pions and kaons. We measure the flux and energy spectrum of the incident neutrinos and compare them with beam simulations. The neutrino-nucleon and anti-neutrino-nucleon differential cross-sections are measured in the range $0.01<x<0.7$, $0.05<y<0.95,10<E_{\nu}<200 \mathrm{GeV}$. We extract the neutrino-nucleon structure functions $F_{2}\left(x, Q^{2}\right), x F_{3}\left(x, Q^{2}\right)$, and $R\left(x, Q^{2}\right)$ and compare these with results from other experiments.
\end{abstract}

To be published in Physics Letters B 


\title{
CHORUS Collaboration
}

\author{
G. Önengüt \\ Çukurova University, Adana, Turkey \\ R. van Dantzig, M. de Jong, R.G.C. Oldeman ${ }^{1}$ \\ NIKHEF, Amsterdam, The Netherlands \\ M. Güler, S. Kama, U. Köse, M. Serin-Zeyrek, P. Tolun \\ METU, Ankara, Turkey \\ M.G. Catanesi, M.T. Muciaccia \\ Università di Bari and INFN, Bari, Italy \\ A. Bülte, K. Winter \\ Humboldt Universität, Berlin, Germany ${ }^{2}$ \\ B. Van de Vyver $^{3,4}$, P. Vilain ${ }^{5}$, G. Wilquet ${ }^{5}$
}

Inter-University Institute for High Energies (ULB-VUB) Brussels, Belgium

B. Saitta

Università di Cagliari and INFN, Cagliari, Italy

E. Di Capua

Università di Ferrara and INFN, Ferrara, Italy

S. Ogawa, H. Shibuya

Toho University, Funabashi, Japan

I.R. Hristova ${ }^{6}$, A. Kayis-Topaksu ${ }^{7}$, T. Kawamura, D. Kolev ${ }^{8}$, M. Litmaath, H. Meinhard, J. Panman,

A. Rozanov ${ }^{9}$, R. Tsenov ${ }^{8}$, J.W.E. Uiterwijk, P. Zucchelli ${ }^{3,10}$

CERN, Geneva, Switzerland

J. Goldberg

Technion, Haifa, Israel

M. Chikawa

Kinki University, Higashiosaka, Japan

J.S. Song, C.S. Yoon

Gyeongsang National University, Jinju, Korea

K. Kodama, N. Ushida

Aichi University of Education, Kariya, Japan

S. Aoki, T. Hara

Kobe University, Kobe, Japan

T. Delbar, D. Favart, G. Grégoire, S. Kalinin, I. Makhlioueva

Université Catholique de Louvain, Louvain-la-Neuve, Belgium

A. Artamonov, P. Gorbunov, V. Khovansky, V. Shamanov, I. Tsukerman

Institute for Theoretical and Experimental Physics, Moscow, Russian Federation

N. Bruski, D. Frekers, D. Rondeshagen, T. Wolff

Westfälische Wilhelms-Universität, Münster, Germany ${ }^{2}$

K. Hoshino, J. Kawada, M. Komatsu, M. Miyanishi, M. Nakamura, T. Nakano, K. Narita, K. Niu,

K. Niwa, N. Nonaka, O. Sato, T. Toshito

Nagoya University, Nagoya, Japan

S. Buontempo, A.G. Cocco, N. D’Ambrosio, G. De Lellis, G. De Rosa, F. Di Capua, G. Fiorillo, A. Marotta, M. Messina, P. Migliozzi, R. Santorelli, L. Scotto Lavina, P. Strolin, V. Tioukov

Università Federico II and INFN, Naples, Italy

T. Okusawa

Osaka City University, Osaka, Japan

U. Dore, P.F. Loverre, L. Ludovici, G. Rosa, R. Santacesaria, A. Satta, F.R. Spada

Università La Sapienza and INFN, Rome, Italy

E. Barbuto, C. Bozza, G. Grella, G. Romano, C. Sirignano, S. Sorrentino

Università di Salerno and INFN, Salerno, Italy

Y. Sato, I. Tezuka

Utsunomiya University, Utsunomiya, Japan 
${ }^{1}$ Now at University of Liverpool, Liverpool, UK.

${ }^{2}$ Supported by the German Bundesministerium fur Bildung und Forschung under contract numbers 05 6BU11P and 05 7MS12P.

${ }^{3}$ Now at SpinX Technologies, Geneva, Switzerland.

${ }^{4}$ Fonds voor Wetenschappelijk Onderzoek, Belgium.

${ }^{5}$ Fonds National de la Recherche Scientifique, Belgium.

${ }^{6}$ Now at DESY, Hamburg.

${ }^{7}$ On leave of absence from Çukurova University, Adana, Turkey.

${ }^{8}$ On leave of absence and at St. Kliment Ohridski University of Sofia, Bulgaria.

${ }^{9}$ Now at CPPM CNRS-IN2P3, Marseille, France.

${ }^{10}$ On leave of absence from INFN, Ferrara, Italy. 
Introduction

High-energy neutrino-nucleon scattering provides a unique view on the inner structure of the nucleon through charged-current interactions that probe the quark-flavour structure in different ways than with charged-lepton scattering. Previous high-statistics measurements of neutrino-nucleon interactions $[1,2]$ have provided strong constraints on the momentum fraction of the nucleon carried by the anti-quarks.

The precision of high-statistics measurements of structure functions is limited by the detailed understanding of the experimental apparatus. Therefore, in large parts of the range covered experimentally, the accuracy is determined by systematic uncertainties rather than statistical errors. The two highstatistics data-sets mentioned above show signifi cant differences in some kinematic regions. Thus additional measurements of the structure functions in a similar kinematic domain are of importance.

The CHORUS detector is well suited to perform measurements of neutrino-nucleon differential cross-sections owing to its high-resolution calorimeter and the large acceptance of the muon spectrometer for neutrino interactions in the calorimeter. In this paper, we describe a measurement of $F_{2}\left(x, Q^{2}\right)$, $x F_{3}\left(x, Q^{2}\right)$, and $R\left(x, Q^{2}\right)$, obtained in an exposure of the lead-scintillator calorimeter of the CHORUS experiment to sign-selected neutrino and anti-neutrino beams. The high-statistics exposure of the CHORUS calorimeter allows us to present the experimental differential cross-section $d^{2} \sigma /(d x d y)$, in different bins of the neutrino energy, with minimal model-dependence and allows QCD analyses to be performed with these data by others.

In charged-current interactions of muon neutrinos with a nucleon, the full kinematics of the interaction can be reconstructed from the measurement of the muon momentum $p_{\mu}$, the angle $\theta_{\mu}$ of the produced muon with respect to the beam axis, and $E_{\text {had }}$, the energy transfer to the hadronic system:

$$
\begin{aligned}
E_{\nu} & =p_{\mu}+E_{\mathrm{had}}, \\
y & =\frac{E_{\mathrm{had}}}{E_{\nu}}, \\
Q^{2} & =4 E_{\nu} p_{\mu} \sin ^{2}\left(\frac{\theta_{\mu}}{2}\right), \\
x & =\frac{Q^{2}}{2 M_{\mathrm{N}} E_{\mathrm{had}}},
\end{aligned}
$$

where $E_{\nu}$ is the energy of the incoming (anti-)neutrino, $y$ is the fractional energy transfer to the hadronic system, $-Q^{2}$ the squared four-momentum transfer, $M_{\mathrm{N}}$ the mass of the nucleon, and $x$ the longitudinal momentum fraction carried by the struck parton. In these relations, the mass of the muon and of the neutrino have been neglected.

In the single- $W$ exchange approximation, the cross-section of neutrino-nucleon scattering can be described in terms of three structure functions that depend on two variables only, namely $F_{1}\left(x, Q^{2}\right)$, $F_{2}\left(x, Q^{2}\right)$, and $x F_{3}\left(x, Q^{2}\right)$ :

$$
\frac{d^{2} \sigma}{d x d y}=\frac{G_{\mathrm{F}}^{2} M_{\mathrm{N}} E_{\nu}}{\pi\left(1+Q^{2} / M_{\mathrm{W}}^{2}\right)^{2}}\left[\frac{y^{2}}{2} 2 x F_{1}+\left(1-y-\frac{M_{\mathrm{N}} x y}{2 E_{\nu}}\right) F_{2} \pm\left(y-\frac{y^{2}}{2}\right) x F_{3}\right],
$$

where $G_{\mathrm{F}}$ is the Fermi coupling constant and $M_{\mathrm{W}}$ the mass of the $\mathrm{W}$ boson.

For isoscalar targets (with an equal number of neutrons and protons), it can be shown that $2 x F_{1}^{\nu}=$ $2 x F_{1}^{\bar{\nu}}$ and $F_{2}^{\nu}=F_{2}^{\bar{\nu}}$, while $x F_{3}^{\bar{\nu}} \neq x F_{3}^{\nu}$ owing to the difference between the strange and the charmed sea. In the remainder of the text we use $x F_{3}=\left(x F_{3}^{\nu}+x F_{3}^{\bar{\nu}}\right) / 2$ and $\Delta x F_{3}=\left(x F_{3}^{\nu}-x F_{3}^{\bar{\nu}}\right) / 2$.

In the quark-parton model, corresponding with leading order and leading twist QCD, neutrinos scatter only off quarks, and for an isoscalar target we fi nd:

$$
\begin{aligned}
2 x F_{1}=F_{2} & =u+\bar{u}+d+\bar{d}+s+\bar{s} \\
x F_{3} & =u-\bar{u}+d-\bar{d}+s-\bar{s},
\end{aligned}
$$

where $u(\bar{u}), d(\bar{d})$, and $s(\bar{s})$ are the $x$ - and $Q^{2}$-dependent up, down, and strange quark (anti-quark) distributions in the proton, respectively. 
Beyond leading order, gluons contribute to the scattering process, and the structure functions can no longer be directly related to the quark densities. Violations of the Callan-Gross relation [3] , $2 x F_{1}=$ $F_{2}$, are usually expressed in terms of $R\left(x, Q^{2}\right)$ :

$$
R=\left(1+\frac{4 M_{\mathrm{N}}^{2} x^{2}}{Q^{2}}\right) \frac{F_{2}}{2 x F_{1}}-1
$$

where $R$ can be interpreted as the ratio of the longitudinal and transverse cross-sections, $\sigma_{L} / \sigma_{T}$.

\section{Experimental parameters}

The CHORUS experiment was primarily designed to search for neutrino oscillations through $\tau^{-}$ production in nuclear emulsions placed in a beam of predominantly muon neutrinos [4]. The data used for the present measurement were taken in the 1998 run, after the nuclear emulsions and the air-core spectrometer had been removed. The available space was used to place nuclear targets used for the measurement of nuclear dependence of the neutrino-nucleon cross-section [5].

Neutrinos are obtained from the decay of pions and kaons, produced in interactions of $450 \mathrm{GeV} / \mathrm{c}$ protons accelerated by the Super Proton Synchrotron at CERN, and extracted every $14.4 \mathrm{~s}$ in two $6 \mathrm{~ms}$ spills containing typically $1.5 \times 10^{13}$ protons each [6]. Two pulsed toroidal magnets downstream of the production target focus either positively charged particles (neutrino mode) or negatively charged particles (anti-neutrino mode). The decay of these charged particles results in a wide-band neutrino beam. A shielding of $225 \mathrm{~m}$ of iron and $144 \mathrm{~m}$ of earth stops most particles, except neutrinos.

The CHORUS detector has been described in detail elsewhere [7]. Here we briefly describe the systems that are most relevant for this analysis: the lead-scintillating-fi bre calorimeter that serves as an active target, and the magnetized-iron spectrometer that measures the muon charge, momentum, and direction.

The CHORUS calorimeter is made of lead and scintillator in a volume ratio of four to one and has both longitudinal and transverse segmentation [8]. Lead-scintillator modules are positioned transversely to the neutrino beam and are read out on both sides by photomultipliers. The fi rst section (EM) consists of $1 \mathrm{~mm}$ thick scintillating fi bres interspersed in lead, bundled together in modules of $4 \mathrm{~cm} \times 4 \mathrm{~cm}$, and arranged in four planes with alternating vertical and horizontal orientation. The second section (HAD1) has the same fi bre/lead structure as EM, but has modules of $8 \mathrm{~cm} \times 8 \mathrm{~cm}$, arranged in fi ve planes. The third section (HAD2) has a readout unit of $10 \mathrm{~cm} \times 10 \mathrm{~cm}$ and is constructed as a sandwich of $4 \mathrm{~mm}$ thick scintillator strips with $16 \mathrm{~mm}$ lead strips. Eleven sets of streamer tube detectors are installed between the calorimeter planes to aid the tracking of muons to the primary vertex. The energy reconstruction and vertex location is performed with a neural net algorithm with two hidden layers that has been trained both on test beam data [9] and on simulated neutrino interactions. The calorimeter energy reconstruction is linear up to $100 \mathrm{GeV}$, at higher energies the linearity is compromised by saturation of the calorimeter ADCs. When the scintillators of the muon spectrometer are included in the shower energy reconstruction, an energy resolution of $20 \%$ is achieved for $8 \mathrm{GeV}$ showers, improving to $12 \%$ for shower energies above $35 \mathrm{GeV}$. The same neural net also determines the longitudinal vertex position with an accuracy of $2.3 \mathrm{~cm}$. A 5\% scale uncertainty is attributed to the calorimeter energy determination. This has been determined from the observed variation of the raw calorimeter response as a function of the interaction depth of testbeam hadrons. The uncertainty on the offset is $150 \mathrm{MeV}$, corresponding to the energy deposit of a minimum-ionizing particle in one plane of the HAD2 section of the calorimeter ${ }^{1)}$. An uncertainty of $2.5 \%$ is attributed to the hadronic energy resolution, corresponding to the statistical uncertainty from the test-beam exposure. The composition of the calorimeter, used in this analysis as a target, is summarized in Table 1.

The muon spectrometer consists of six magnetized iron disks with an outer diameter of $375 \mathrm{~cm}$ and an average strength of the toroidal fi eld of $1.7 \mathrm{~T}$. The disks are interspersed with scintillating strips used for measuring the energy leakage of showers not fully contained in the calorimeter. Drift chambers and streamer tubes are positioned between the magnets to follow the muon trajectory and to measure its curvature in the magnetic fi eld. During neutrino running, negatively charged muons are bent toward

\footnotetext{
1) In the EM and HAD1 calorimeter sections the energy deposit is $50 \mathrm{MeV}$ and $100 \mathrm{MeV}$ per plane, respectively.
} 
Table 1: Material composition of the calorimeter. The column 'other' refers to scintillator material for EM, HAD1, and HAD2, and to PVC and aluminium for the streamer tubes.

\begin{tabular}{lrrrrrrr}
\hline & Planes & $\begin{array}{r}\text { Density } \\
\left(\mathrm{g} \mathrm{cm}^{-2}\right)\end{array}$ & $\begin{array}{r}\text { Lead } \\
\left(\mathrm{g} \mathrm{cm}^{-2}\right)\end{array}$ & $\begin{array}{r}\text { Iron } \\
\left(\mathrm{g} \mathrm{cm}^{-2}\right)\end{array}$ & $\begin{array}{r}\text { Other } \\
\left(\mathrm{g} \mathrm{cm}^{-2}\right)\end{array}$ & $x / x_{0}$ & $x / \lambda_{I}$ \\
\hline EM & 4 & 37.33 & 34.67 & 1.89 & 0.77 & 5.60 & 0.203 \\
HAD1 & 5 & 76.09 & 71.36 & 3.15 & 1.58 & 11.47 & 0.412 \\
HAD2 & 5 & 92.23 & 87.93 & 2.36 & 1.94 & 14.02 & 0.495 \\
Streamer tubes & 22 & 0.77 & & & 0.77 & 0.02 & 0.009 \\
\hline Total & & 1007.86 & 935.13 & 35.11 & 37.62 & 150.29 & 5.545 \\
\hline
\end{tabular}

the centre of the magnets and positively charged muons are bent outward; for anti-neutrino running the polarity is inverted. The muon momentum is determined from the curvature of its trajectory in the toroidal magnetic fi eld. The momentum resolution varies from $15 \%$ [10] in the $12-28 \mathrm{GeV} / c$ interval to $19 \%$ [7] at $70 \mathrm{GeV} / c$, as measured with test-beam muons, and is well reproduced in simulations. A $2.5 \%$ scale uncertainty is attributed to the momentum measurement due to imperfections of magnetic fi eld measurements, and the uncertainty on the offset is $150 \mathrm{MeV} / c$.

For the 1998 run, the CHORUS trigger system [11] was adjusted to record charged-current interactions with minimal bias. Three or more calorimeter planes with hits in the central parts of the HAD1 and HAD2 sections are required, in coincidence with activity in at least two of the four most upstream spectrometer magnets. Neutrino interactions upstream of the detector are vetoed by means of a large plane of veto scintillators $4 \mathrm{~m}$ in front of the calorimeter. The data acquisition system [12] makes it possible to record up to 16 interactions for every accelerator cycle.

\section{$3 \quad$ Analysis procedure}

The accuracy of detailed simulations of wide-band neutrino beams is insuffi cient to be used directly for the flux normalization. Therefore, we fi rst measure the neutrino flux and its energy spectrum from the data. In a second step, the differential neutrino-nucleon cross-section is measured as a function of $x, y$, and $E_{\nu}$ for both neutrinos and anti-neutrinos. Finally, the measured differential cross-sections are used to extract the structure functions $F_{2}\left(x, Q^{2}\right), x F_{3}\left(x, Q^{2}\right)$, and $R\left(x, Q^{2}\right)$. An overall scale factor is applied to the results to reproduce the neutrino and anti-neutrino total cross-sections available in the the literature [13].

The event selection of charged-current interactions is based on the presence of a muon with a momentum of at least $5 \mathrm{GeV} / c$ and an angle $\theta_{\mu}$ with respect to the neutrino beam direction of less than $300 \mathrm{mrad}$. To ensure a high purity, the muon candidate is required to penetrate at least four spectrometer magnets. The interaction vertex is required to be reconstructed in the central part of the downstream half of the EM sector or the HAD1 sector. Quasi-elastic interactions and most resonance production are rejected by requiring a minimum hadronic energy of $4 \mathrm{GeV}$; and a maximum of $100 \mathrm{GeV}$ is applied to reject events in the non-linear regime of the calorimeter energy measurement. A summary of the number of events surviving these criteria is shown in Table 2.

A parametrized simulation is used to correct for the fi nite acceptance and detector smearing. The differential cross-section is modelled based on the GRV98LO parton distribution functions (PDF) [14]. The ratio of longitudinal to transverse structure function, $R\left(x, Q^{2}\right)$, is modelled after the SLACR90 parametrization [15], and the nuclear dependence follows the parametrization of Ref. [16]. Target mass corrections are incorporated by evaluating the PDF at the Nachtmann variable $\xi$, which is defi ned as $\xi=2 x /\left(1+\sqrt{1+4 M_{\mathrm{N}}^{2} x^{2} / Q^{2}}\right)$ [17]. Suppression of the cross-section due to charm production is taken into account by replacing $x$ by the slow rescaling variable $x\left(1+m_{c}^{2} / Q^{2}\right)$ [18], where we use $m_{c}=1.31 \mathrm{GeV} / c^{2}$ [19]. Radiative corrections are applied according to the prescription of Bardin [20]. We evaluate the uncertainty of the radiative corrections from the full difference between using GRV98 or CCFR parton distribution functions as input. Finally, a phenomenological correction $c\left(x, Q^{2}\right)$ is applied to the cross-section model to obtain a better description of the measured differential cross-sections at low $Q^{2}$ [21]. This correction is obtained iteratively by comparing the measured cross-sections to the 
Table 2: Numbers of events obtained for the beam flux analysis. $y_{\mathrm{vtx}}$ and $x_{\mathrm{vtx}}$ are the horizontal coordinate perpendicular to the beam and in the beam direction, respectively, while $z_{\mathrm{vtx}}^{*}$ is the coordinate perpendicular to $y_{\mathrm{vtx}}$ and the beam axis.

\begin{tabular}{lcccc}
\hline & neutrino mode & \multicolumn{2}{c}{ anti-neutrino mode } \\
\hline Run selection & $3,631,967$ & \multicolumn{2}{c}{$1,031,741$} \\
Reconstructed muon & $3,105,332$ & \multicolumn{2}{c}{859,309} \\
$x_{\mathrm{vtx}}$ in plane $2-7$ & $1,857,352$ & \multicolumn{2}{c}{523,609} \\
$-90 \leq y_{\mathrm{vtx}}, z_{\mathrm{vtx}}^{*} \leq 90 \mathrm{~cm}$ & $1,252,289$ & \multicolumn{2}{c}{335,927} \\
\hline \multicolumn{1}{c}{$\nu$} & $\bar{\nu}$ & $\nu$ & $\bar{\nu}$ \\
\hline Muon charge & \multicolumn{1}{c}{$1,224,051$} & 28,238 & 83,769 & 252,158 \\
$p_{\mu} \geq 5 \mathrm{GeV} / c$ & $1,161,238$ & 26,599 & 80,695 & 243,317 \\
$4 \leq E_{\mathrm{had}} \leq 100 \mathrm{GeV}$ & 898,522 & 17,925 & 64,538 & 147,410 \\
$\theta_{\mu} \leq 300 \mathrm{mrad}$ & 882,132 & 17,900 & 64,348 & 146,650 \\
$10 \leq E_{\nu} \leq 200 \mathrm{GeV}$ & 870,252 & 17,142 & 62,005 & 145,816 \\
\hline
\end{tabular}

model until they agree. The magnitude of this correction can reach up to $30 \%$ for $Q^{2}<1 \mathrm{GeV}^{2} / c^{2}$, but is negligible for $Q^{2}>5 \mathrm{GeV}^{2} / c^{2}$. We apply a systematic uncertainty due to these corrections by evaluating the difference in acceptance between the default cross-section model and a model without phenomenological corrections.

The detector smearing and muon reconstruction effi ciency have been evaluated making use of a GEANT3 [22] simulation incorporating a detailed description of the detector geometry. We fi nd that the detector response depends predominantly on the muon momentum, the hadronic energy, and the longitudinal vertex position. The events are binned in these three variables, and the resolutions and effi ciency are determined for each combination. While most detector resolutions behave as expected from test-beam data, some degradation of the muon-momentum resolution and reconstruction effi ciency is observed for neutrino interactions with large shower leakage into the muon spectrometer. We have compared the hit multiplicity between data and simulation of events with a high energy hadronic shower and a vertex position in the downstream part of the detector. We fi nd that the multiplicities are consistent between data and the full detector simulation. An uncertainty of 5\% is attributed to the fraction of events not reconstructed. This value was estimated by observing the difference of the results of the simplifi ed simulation and the full simulation of the detector response.

To ensure that the reported values refer to the centre of each bin and not to the average over the bin, bin-centring corrections are applied to the data. This correction is determined as the ratio between the beam flux or cross-section at the centre of the bin and the average value over the bin, calculated from the beam flux and cross-section models. For most bins, the correction is well below $10 \%$.

Systematic uncertainties are evaluated by repeating the analysis with either a $1 \sigma$ shift or taking the difference between two models. The sources of systematic uncertainties and the applied variations are listed in Table 3.

A more detailed description of the analysis procedure can be found in Refs. [21, 23].

\section{Beam flux measurement}

For the beam flux measurement the data are binned in 10 bins of $E_{\nu}$ with variable bin size, from $10 \mathrm{GeV}$ to $200 \mathrm{GeV}$. After acceptance corrections, the yield is normalized to the thickness of the fi ducial volume; to the number of protons delivered on target, measured with beam current transformers (BCT) and corrected for the detector dead-time; and to the total neutrino-nucleon cross-section [13]:

$$
\begin{aligned}
& \sigma_{0}^{\nu N} \equiv \sigma^{\nu N} / E_{\nu}=(0.677 \pm 0.014) \times 10^{-38} \mathrm{~cm}^{2} \mathrm{GeV}^{-1} \\
& \sigma_{0}^{\bar{\nu} N} \equiv \sigma^{\bar{\nu} N} / E_{\bar{\nu}}=(0.500 \pm 0.007) \sigma_{0}^{\nu N}
\end{aligned}
$$

In the energy range relevant for this analysis, the non-linearity of the neutrino-nucleon crosssection is smaller than $1 \%$ per $100 \mathrm{GeV}$ and the anti-neutrino to neutrino cross-section less than $0.5 \%$ 
Table 3: Overview of the variations applied to evaluate systematic uncertainties. The uncertainty from the calibration of the beam current transformer (BCT) affects the beam flux measurement, but cancels for the cross-section and structure function measurements.

\begin{tabular}{lc}
\hline Error source & Variation \\
\hline Hadronic energy scale & $5 \%$ \\
Hadronic energy offset & $150 \mathrm{MeV}$ \\
Hadronic energy resolution & $2.5 \%$ \\
Muon momentum scale & $2.5 \%$ \\
Muon momentum offset & $150 \mathrm{MeV} / c$ \\
Reconstruction ineffi ciency & $\pm 5 \%$ \\
Total $\nu$-nucleon cross-section & $2.1 \%$ \\
Ratio of $\bar{\nu}$-nucleon and $\nu$-nucleon cross-section & $1.4 \%$ \\
Non-linearity of the $\nu$-nucleon cross-section & $1 \% / 100 \mathrm{GeV}$ \\
Non-linearity of the $\bar{\nu}$-nucleon to $\nu$-nucleon cross-section ratio & $0.5 \% / 100 \mathrm{GeV}$ \\
Acceptance corrections & with/without pheno- \\
& menological corrections \\
Radiative corrections & $\mathrm{CCFR} / \mathrm{GRV} 98$ \\
Callan-Gross violation & $R \pm 20 \%$ \\
Strange sea & $\Delta x F_{3} \pm 20 \%$ \\
BCT calibration (beam flux only) & $2 \%$ \\
\hline
\end{tabular}

per $100 \mathrm{GeV}$. A correction for the neutron excess in the target is applied to the flux measurement such that the measured flux is defi ned as the rate of interactions on an isoscalar target. The size of this correction is about $-6 \%(+6 \%)$ for the neutrino (anti-neutrino) flux.

The results are shown in Fig. 1 and are compared with the prediction from the CHORUS beam simulation GBEAM $[24,25]$, a GEANT3 simulation of the neutrino beam, using the FLUKA [26] package for the primary hadronic interactions. We fi nd that the energy spectra are well predicted by the beam simulations, but the absolute yields are overestimated by about $10 \%$ ( $20 \%$ for anti-neutrinos). Our measurements are in agreement with the NOMAD measurements of the same beam [27]. For the Monte Carlo generation, the neutrino spectra are fi tted to a smooth function:

$$
N\left(E_{\nu}\right)=\exp \left(p_{1}-\frac{E_{\nu}}{p_{2}}-\frac{p_{3}}{E_{\nu}}\right)+\exp \left(p_{4}-\frac{E_{\nu}}{p_{5}}-\frac{p_{6}}{E_{\nu}}\right),
$$

where the fi rst term represents the soft component from pion decay and the second term represents the hard component from kaon decay.

\section{$5 \quad$ Measurement of the differential cross-sections}

The differential cross-section measurement is normalized to the total neutrino-nucleon crosssection and is thus not affected by the discrepancies between the beam simulation and the beam flux measurement. For the determination of the differential cross-sections, the neutrino and anti-neutrino data are counted in bins of $x, y, E_{\nu}$. Only the focused components of the neutrino and anti-neutrino beam exposures are used. The data sample and the selection criteria are identical to those used for the beam flux measurement. The cuts on $p_{\mu}, \theta_{\mu}$, and $E_{\text {had }}$ restrict the kinematical domain of the differential crosssection measurement. Only those bins that are fully contained within the kinematical cuts are accepted for the analysis.

The differential cross-section is determined by the ratio of events $N\left(E_{i}, x_{j}, y_{k}\right)$ to the total number of neutrino interactions in the same energy bin:

$$
\frac{1}{E_{\nu}} \frac{d^{2} \sigma}{d x d y}=\frac{\sigma_{\mathrm{tot}}}{E_{\nu}} \frac{N\left(E_{i}, x_{j}, y_{k}\right)}{N\left(E_{i}\right) \Delta x_{j} \Delta y_{k}}
$$

where $\Delta x_{j}$ and $\Delta y_{k}$ are the sizes of the bins in $x$ and $y$ respectively, and $\sigma_{\text {tot }}$ is the total $\nu N$ cross-section according to Eq 9-10, corrected for the neutron excess in the target. 
Smearing and effi ciency corrections are calculated bin-by-bin by taking the ratio of the number of events generated with the true value in a bin to the number of events reconstructed with the smeared values in that bin. From an event migration study we fi nd that $64 \%$ of the events is reconstructed in the right energy bin, $41 \%$ in the right $x$-bin and $67 \%$ in the right $y$-bin. The results of the differential cross-section measurement are shown in Fig. 2. The differential cross-section has not been corrected for the non-isoscalarity of the target and thus refers to the cross-section on the material of the CHORUS calorimeter, which is $93 \%$ lead, $3.5 \%$ iron and $3.5 \%$ other materials.

The differential cross-section of the model is shown as a curve in Fig. 2. The agreement between the measurement and the model validates the use of the model for the calculation of the acceptance and smearing corrections applied to the data.

Several characteristic properties of the differential neutrino-nucleon cross-section can be observed in Fig. 2. The numerical values of all measurements reported in the fi gures are available in Ref. [28]. At low $x$, it is expected that the cross-section is dominated by scattering off sea quarks, and indeed the relative difference between the measured neutrino and anti-neutrino cross-sections is small. Scaling violations, predicted by QCD, are also visible: at low $x$ the cross-section increases with $E_{\nu}$, while at high $x$ the cross-section decreases with $E_{\nu}$.

\section{Structure function extraction}

To extract structure functions corresponding to an isoscalar target, the cross-section data are corrected for radiative effects and for the $9.7 \%$ excess of neutrons in the target, based on the difference between the $u$ and $d(\bar{u}$ and $\bar{d})$ distributions in the GRV98LO parton distributions. Isoscalarity corrections are most signifi cant at high $x$, but never exceed $15 \%$. The cross-section points are grouped in bins of $\left(x, Q^{2}\right)$. The binning in $x$ is the same as used for the differential cross-section measurement. The binning in $Q^{2}$ is equidistant in $\log \left(Q^{2}\right)$ and divides the range $0.1-100 \mathrm{GeV}^{2} / c^{2}$ into 15 bins. The low- $Q^{2}$ bins have more entries at low $y$, while the high- $Q^{2}$ bins have more entries at high $y$. A correction is applied to shift the cross-section points to the centre of each $Q^{2}$ bin.

The $y$ dependence of both neutrino and anti-neutrino data is then used to extract the structure functions $F_{2}\left(x, Q^{2}\right), x F_{3}\left(x, Q^{2}\right)$, and $R\left(x, Q^{2}\right)$, by applying a linear 3-parameter fi $\mathrm{t}$ in each $\left(x, Q^{2}\right)$ bin according to Eqs. (5) and (8). The results for $R\left(x, Q^{2}\right)$ are shown in Fig. 3 and compared with the SLACR90 parametrization.

Since the results on $R\left(x, Q^{2}\right)$ are in agreement with the more precise measurements from chargedlepton scattering, $R$ is fi xed to the SLACR90 parametrization to improve the precision and extend kinematic range for the extraction of the structure functions $F_{2}\left(x, Q^{2}\right)$ and $x F_{3}\left(x, Q^{2}\right)$. The results are shown in Figs. 4 and 5 and compared with the results from the CCFR [1] and CDHSW [2] experiments. For $x F_{3}\left(x, Q^{2}\right)$ our results are in agreement with both experiments. For $F_{2}\left(x, Q^{2}\right)$, where the two other experiments disagree, our data favour the CCFR results and confi rm the expectation that the difference between the nucleon structure functions of lead and iron is small. Preliminary $\mathrm{NuTeV}$ results [29] are in agreement with the CCFR data for low and intermediate $x$ but indicate higher values of $F_{2}$ for $x \geq 0.55$. Due to the relatively large systematic uncertainty at high $x$ and the difference in target material, we are not able to distinguish between the two.

\section{Conclusion}

Using data from the 1998 CHORUS run, we measure the muon neutrino and anti-neutrino beam flux and energy spectra. Detailed beam simulations give a good prediction of the energy spectra but overestimate the absolute flux by $10 \%$ for the neutrino component and by $20 \%$ for the anti-neutrino component. We provide the fi rst high-statistics measurement of the differential (anti-)neutrino crosssections on a target predominantly made of lead as a function of the neutrino energy and the Björken variables $x$ and $y$. The differential cross-sections are used to extract the structure functions $R\left(x, Q^{2}\right)$, $F_{2}\left(x, Q^{2}\right)$, and $x F_{3}\left(x, Q^{2}\right)$. Our data on $R$ are in agreement with SLAC data and our measurement of $x F_{3}$ agrees with both CCFR and CDHSW. Our measurement of $F_{2}$ favours the CCFR data over CDHSW. 


\section{Acknowledgements}

We gratefully acknowledge the help and support of the neutrino-beam staff and of the numerous technical collaborators who contributed to the detector construction and operation. The experiment has been made possible by grants from the Institut Interuniversitaire des Sciences Nucléaires and the Interuniversitair Instituut voor Kernwetenschappen (Belgium); the Israel Science Foundation (grant 328/94) and the Technion Vice President Fund for the Promotion of Research (Israel); CERN (Geneva, Switzerland); the German Bundesministerium für Bildung und Forschung (Germany); the Institute of Theoretical and Experimental Physics (Moscow, Russia); the Istituto Nazionale di Fisica Nucleare (Italy); the Promotion and Mutual Aid Corporation for Private Schools of Japan and Japan Society for the Promotion of Science (Japan); the Korea Research Foundation Grant KRF-2003-005-C00014 (Republic of Korea); the Foundation for Fundamental Research on Matter FOM and the National Scientifi c Research Organisation NWO (The Netherlands); and the Scientifi c and Technical Research Council of Turkey (Turkey). We gratefully acknowledge their support.

\section{References}

[1] W. G. Seligman et al., CCFR Collaboration, Phys. Rev. Lett. 79 (1997) 1213.

[2] J. P. Berge et al., CDHSW Collaboration, Z. Phys. C 49 (1991) 187.

[3] C. G. Callan and D. J. Gross, Phys. Rev. Lett. 22 (1969) 156.

[4] E. Eskut et al., CHORUS Collaboration, Phys. Lett. B 497 (2001) 8.

[5] A. Kayis-Topaksu et al., CHORUS Collaboration, Eur. Phys. J. C 30 (2003) 159.

[6] G. Acquistapace et al., CERN-ECP 95-14, 1995.

[7] E. Eskut et al., CHORUS Collaboration, Nucl. Instrum. Meth. A 401 (1997) 7.

[8] S. Buontempo et al., Nucl. Phys. Proc. Suppl. B 54 (1997) 198.

[9] E. Di Capua et al., Nucl. Instrum. Meth. A 378 (1996) 221.

[10] A. Artamonov and P. Gorbunov, CHORUS Internal Note 97029, 23 February 1998, updated on 12 March 1999.

http://choruswww. cern.ch/Publications/Notes/spec1munew.pdf

[11] M. G. van Beuzekom et al., CHORUS Collaboration, Nucl. Instrum. Meth. A 427 (1999) 587.

[12] A. Artamonov et al., Nucl. Instrum. Meth. A 479 (2002) 412.

[13] J. M. Conrad, M. H. Shaevitz and T. Bolton, Rev. Mod. Phys. 70 (1998) 1341.

[14] M. Glück, E. Reya and A. Vogt, Eur. Phys. J. C 5 (1998) 461.

[15] L. W. Whitlow, S. Rock, A. Bodek, E. M. Riordan and S. Dasu, Phys. Lett. B 250 (1990) 193.

[16] J. Gomez et al., Phys. Rev. D 49 (1994) 4348.

[17] O. Nachtmann, Nucl. Phys. B 78 (1974) 455.

[18] R. M. Barnett, Phys. Rev. Lett. 36 (1976) 1163. H. Georgi and H. D. Politzer, Phys. Rev. D 14 (1976) 1829.

[19] S. A. Rabinowitz et al., Phys. Rev. Lett. 70 (1993) 134.

[20] D. Y. Bardin and V. A. Dokuchaeva, JINR-E2-86-260, 1986.

[21] R. G. C. Oldeman, Ph.D. thesis, University of Amsterdam, 2000.

[22] R. Brun and F. Carminati CERN Program Library Long Writeup W5013, 1993.

[23] S. Kama, M.Sc. thesis, Middle East Technical University, Ankara, 2005.

[24] S. Sorrentino, Ph.D. thesis, Salerno University, 1999.

[25] I. Tsukerman, CHORUS Collaboration, Nucl. Phys. Proc. Suppl. 112 (2002) 177.

[26] A. Fasso, A. Ferrari, P. R. Sala and J. Ranft, SLAC-REPRINT-2000-117 Prepared for International Conference on Advanced Monte Carlo for Radiation Physics, Particle Transport Simulation and Applications (MC 2000), Lisbon, Portugal, 23-26 Oct 2000.

[27] P. Astier et al., NOMAD Collaboration, Nucl. Instrum. Meth. A 515 (2003) 800.

[28] Full listings of the measured cross-sections and structure functions, including the effect of individual systematic shifts, are available in ASCII format at http://choruswww. cern. ch/Publications/DIS-data

[29] M. Tzanov et al. [NuTeV Collaboration], arXiv:hep-ex/0509010. 

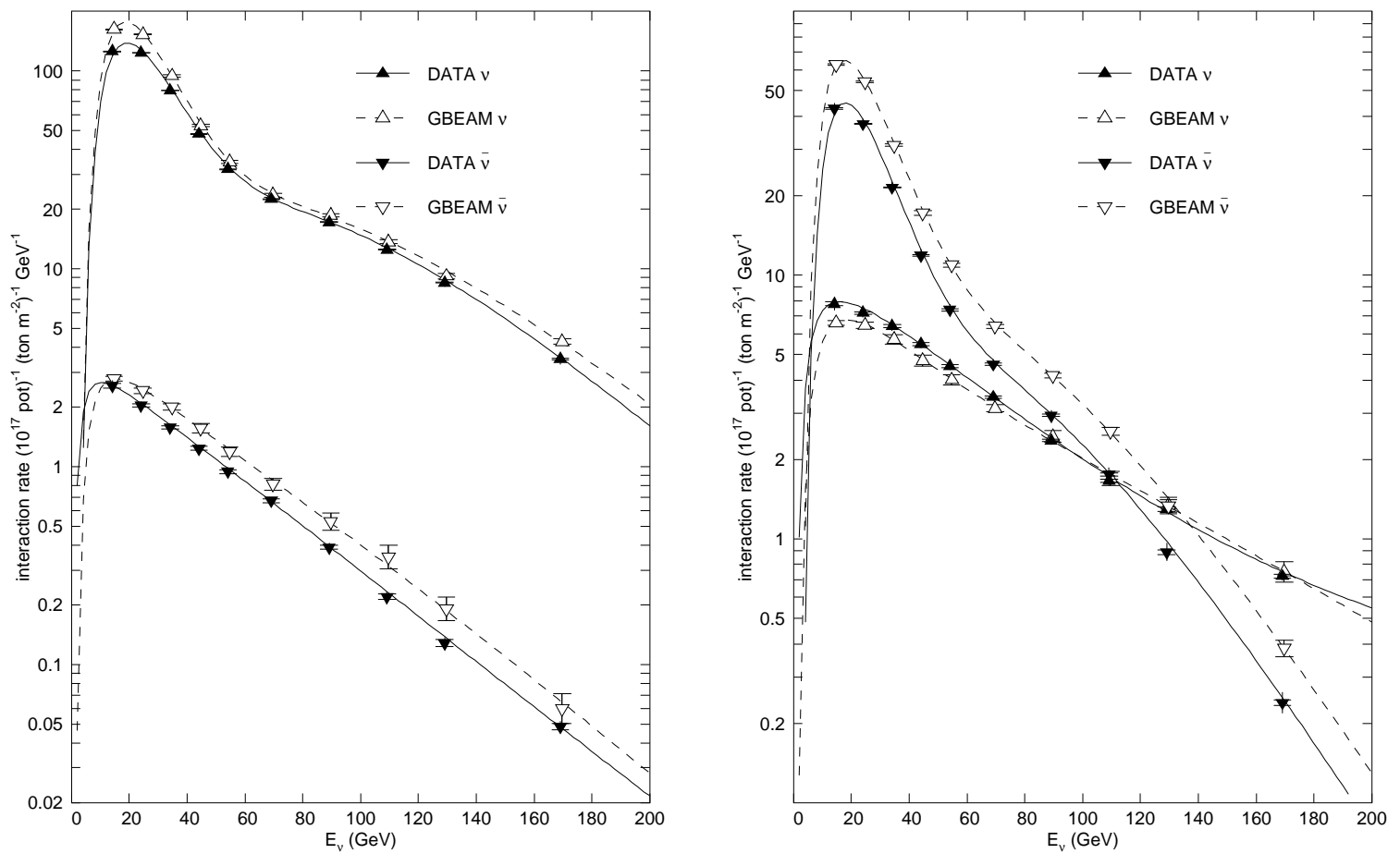

Figure 1: Measurements of the neutrino beam fluxes for neutrino beam (left) and anti-neutrino beam (right). The measurements (solid triangles) are compared with the GBEAM simulation (open triangles). The solid (dashed) lines indicate fi ts to the data (simulation) described in the text. 


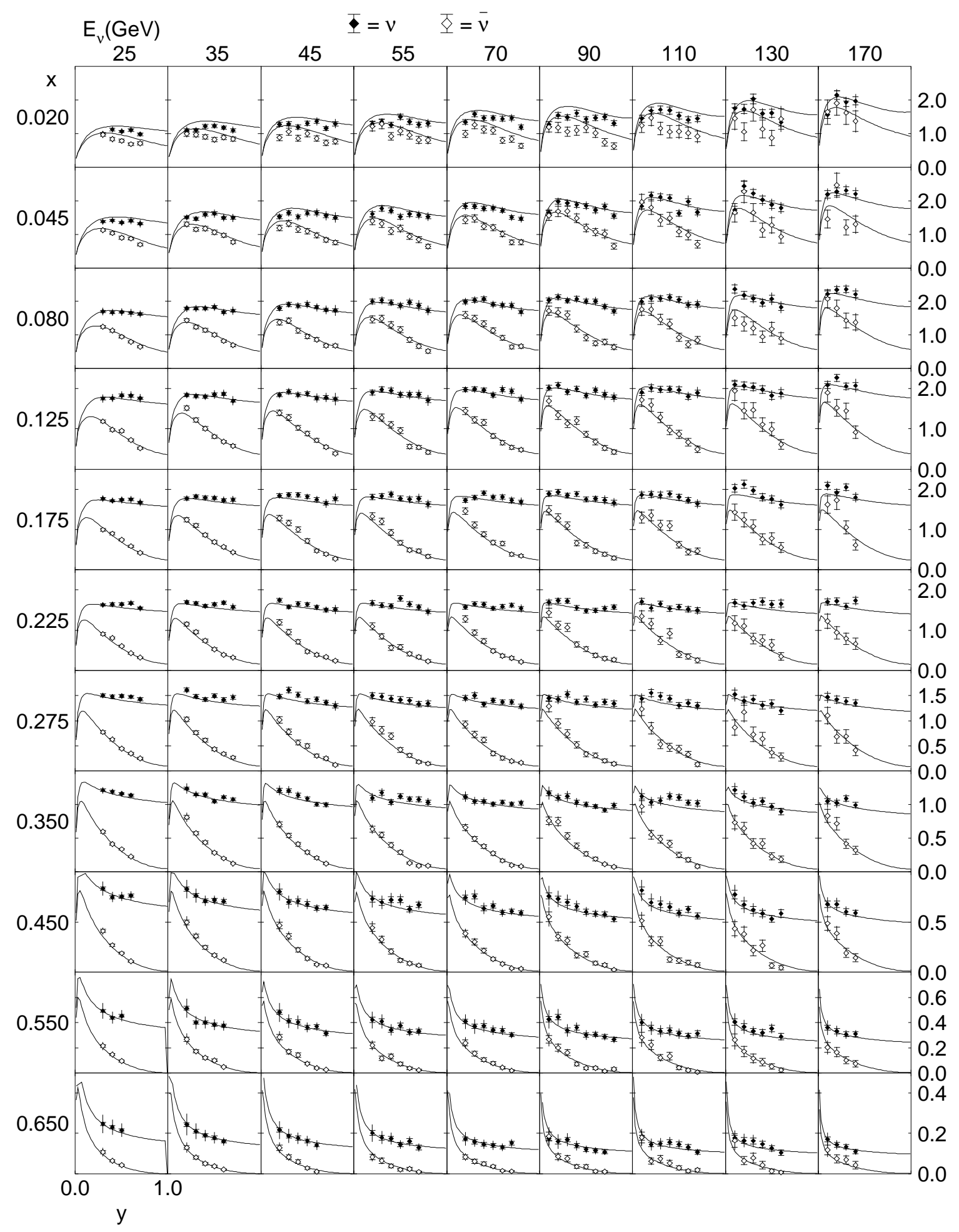

Figure 2: Measured differential cross-sections of neutrino-nucleon scattering on the CHORUS calorimeter. Points are from data where the inner bar represents the statistical uncertainty and the outer error bar the quadratic sum of statistical and systematic uncertainties. The curves indicate the cross-section model described in the text. Numerical values of these measurements are available in Ref. [28]. 

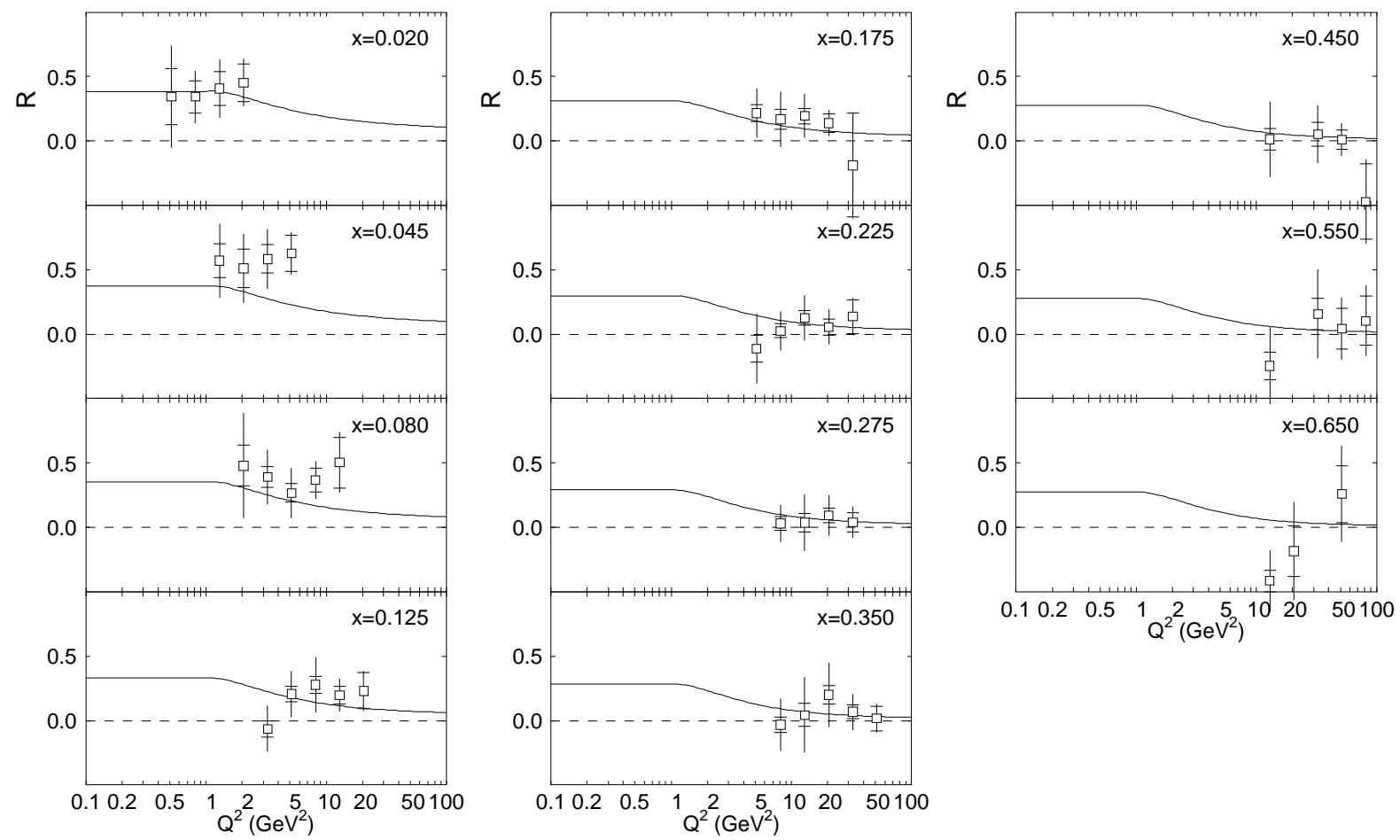

Figure 3: Result of the extraction of the structure function $R\left(x, Q^{2}\right)$. Points are from data where the inner bar represents the statistical uncertainty and the outer error bar the quadratic sum of statistical and systematic uncertainties. The curves indicate the SLACR90 parametrization. Numerical values of these measurements are available in Ref. [28]. 


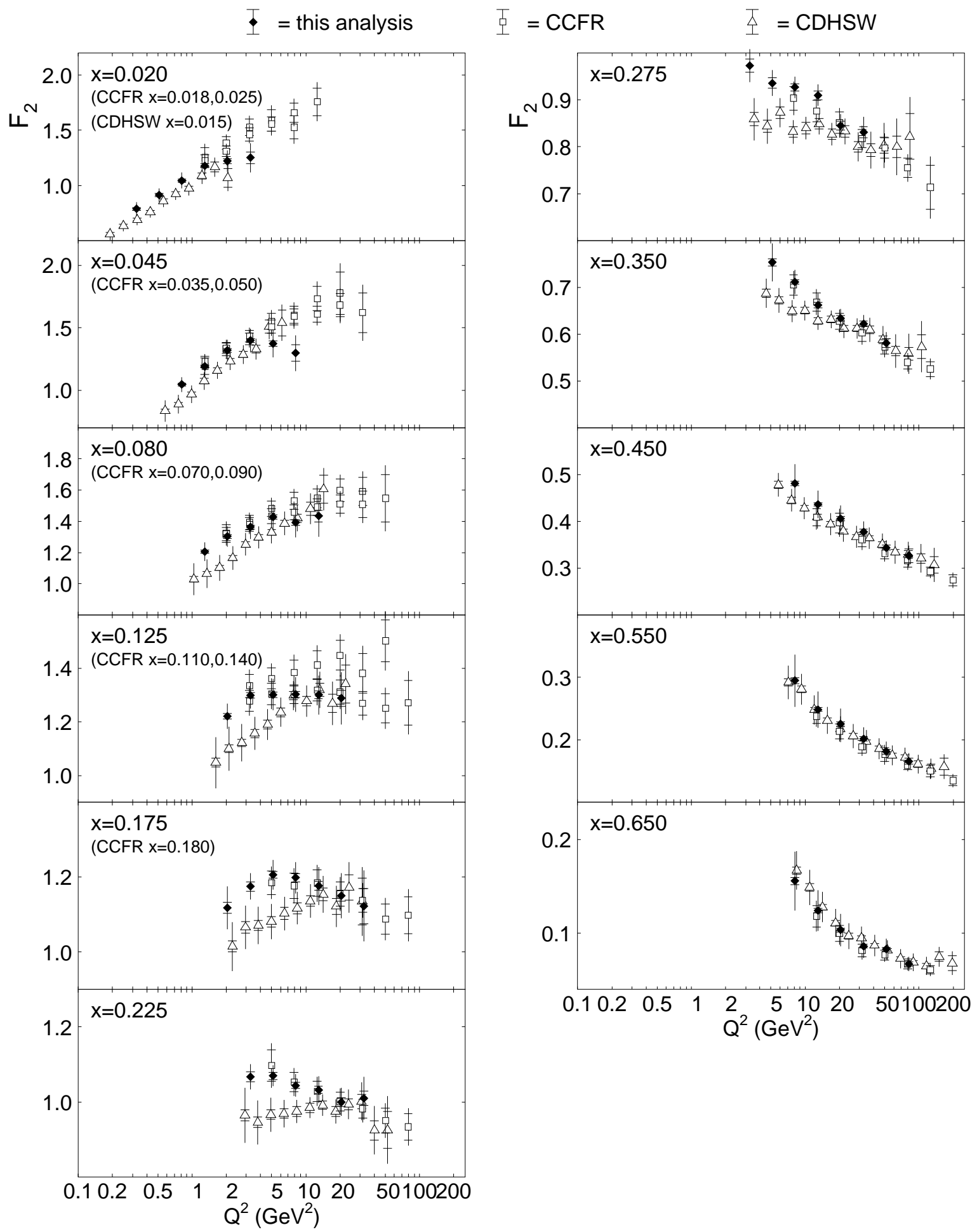

Figure 4: Comparison of our $F_{2}\left(x, Q^{2}\right)$ results with measurements from CCFR and CDHSW. The inner bars represent the statistical uncertainties and the outer bars the quadratic sum of statistical and systematic uncertainties. Numerical values of these measurements are available in Ref. [28]. 


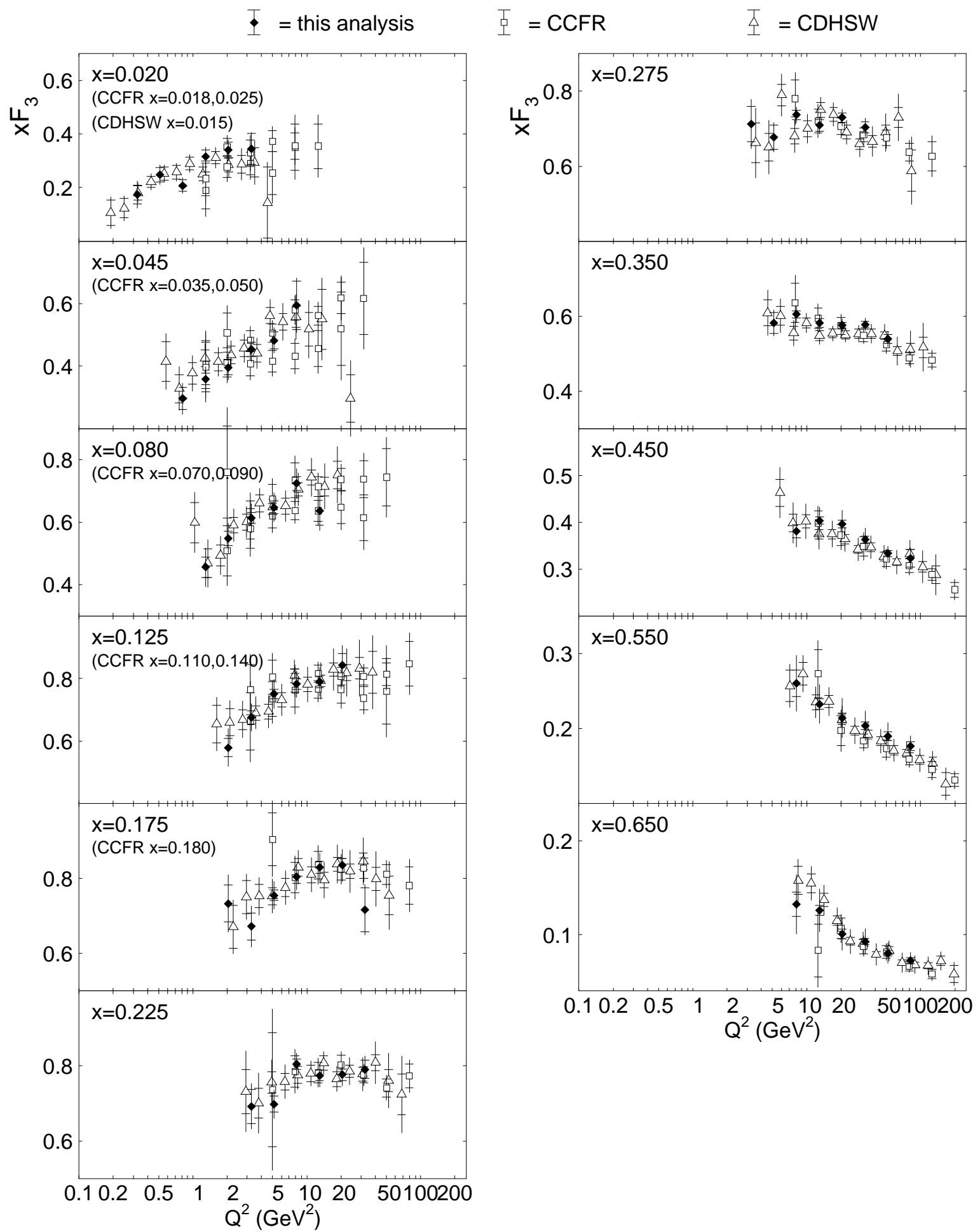

Figure 5: Comparison of our $x F_{3}\left(x, Q^{2}\right)$ results with measurements from CCFR and CDHSW. The inner bars represent the statistical uncertainties and the outer bars the quadratic sum of statistical and systematic uncertainties. Numerical values of these measurements are available in Ref. [28]. 